Published in final edited form as:

Nat Chem Biol. 2010 August ; 6(8): 595-601. doi:10.1038/nchembio.391.

\title{
The MCL-1 BH3 Helix is an Exclusive MCL-1 inhibitor and Apoptosis Sensitizer
}

\author{
Michelle L. Stewart ${ }^{1,2}$, Emiko Fire ${ }^{3}$, Amy E. Keating ${ }^{3}$, and Loren D. Walensky ${ }^{1,2,{ }^{*}}$ \\ ${ }^{1}$ Department of Pediatric Oncology, Dana-Farber Cancer Institute and Children's Hospital \\ Boston, Harvard Medical School, Boston, Massachusetts 02115 \\ 2 Program in Cancer Chemical Biology, Dana-Farber Cancer Institute, Boston, Massachusetts \\ 02115
}

${ }^{3}$ Department of Biology, Massachusetts Institute of Technology, Boston, Massachusetts 02139

\section{Abstract}

The development of selective inhibitors for discrete anti-apoptotic BCL-2 family proteins implicated in pathologic cell survival remains a formidable but pressing challenge. Precisely tailored compounds would serve as molecular probes and targeted therapies to study and treat human diseases driven by specific anti-apoptotic blockades. In particular, MCL-1 has emerged as a major resistance factor in human cancer. By screening a library of Stabilized Alpha-Helix of BCL-2 domains (SAHBs), we determined that the MCL-1 BH3 helix is itself a potent and exclusive MCL-1 inhibitor. X-ray crystallography and mutagenesis studies defined key binding and specificity determinants, including the capacity to harness the hydrocarbon staple to optimize affinity while preserving selectivity. MCL-1 SAHB directly targets MCL-1, neutralizes its inhibitory interaction with pro-apoptotic BAK, and sensitizes cancer cells to caspase-dependent apoptosis. By leveraging nature's solution to ligand selectivity, we generated an MCL-1-specific agent that defines the structural and functional features of targeted MCL-1 inhibition.

Beginning with the discovery of BCL-2 at the t14;18 chromosomal breakpoint of follicular lymphoma ${ }^{1}$, the anti-apoptotic members of the BCL-2 family have emerged as key pathogenic proteins in human diseases characterized by unchecked cellular survival, such as cancer and autoimmunity ${ }^{2}$. A series of anti-apoptotic proteins including BCL-2, BCL- $\mathrm{X}_{\mathrm{L}}$, BCL-w, MCL-1, and BFL1/A1 promote cellular survival by trapping the critical apoptosisinducing BCL-2 homology domain 3 (BH3) a-helix of pro-apoptotic BCL-2 family members ${ }^{3}$. Cancer cells exploit this physiologic survival mechanism through anti-apoptotic

Users may view, print, copy, download and text and data- mine the content in such documents, for the purposes of academic research, subject always to the full Conditions of use: http://www.nature.com/authors/editorial_policies/license.html\#terms

To whom correspondence should be addressed: Loren D. Walensky, M.D., Ph.D,. Dana-Farber Cancer Institute, 44 Binney Street, Mayer 664, Boston, MA 02115, Office: 617-632-6307, Fax: 617-582-8240, Loren_Walensky@dfci.harvard.edu. AUTHOR CONTRIBUTIONS

M.L.S. and L.D.W. designed, synthesized, and characterized SAHB compounds for biochemical, structural, and cellular analyses. M.L.S. performed the x-ray crystallography experiments with E.F. in the laboratory of A.E.K., who supervised the structural analyses. M.L.S. conducted all biochemical and cellular analyses, with guidance from L.D.W. L.D.W. and M.L.S. wrote the manuscript, which was reviewed and edited by E.F. and A.E.K.

Loren Walensky is a scientific advisor board member and consultant for Aileron Therapeutics. 
protein overexpression, establishing an apoptotic blockade that secures their immortality. To overcome this potentially fatal resistance mechanism, a pharmacologic quest is underway to develop targeted therapies that bind and block BCL-2 family survival proteins.

Anti-apoptotic proteins contain a hydrophobic binding pocket on their surface that engages BH3 a-helices ${ }^{3},{ }^{4}$. Because nature's solution to anti-apoptotic targeting involves selective interactions between $\mathrm{BH} 3$ death domains and anti-apoptotic pockets ${ }^{5},{ }^{6}$, molecular mimicry of the BH3 a-helix has formed the basis for developing small molecule inhibitors of antiapoptotic proteins ${ }^{7}{ }^{9}$. Promising compounds undergoing clinical evaluation, such as ABT- $263^{10}$, obatoclax ${ }^{8}$, and AT- $101^{11}$, each target three or more anti-apoptotic proteins. The development of precise inhibitors that target individual anti-apoptotic proteins remains a significant challenge due to the subtle differences among BH3-binding pockets. Reminiscent of the long-term goals in kinase therapeutics, anti-apoptotic inhibitors with tailored specificity would provide finely-tuned therapies to treat distinct diseases while potentially avoiding unwanted side-effects. In addition, such compounds would serve as invaluable research tools to dissect the differential biological functions of anti-apoptotic proteins.

The specificity of anti-apoptotic proteins for $\mathrm{BH} 3$ domains is conferred by the topography of the canonical binding groove and the distinctive amino acid composition of the interacting BH3 helix. Whereas some BH3 domains, such as that of pro-apoptotic BIM, can tightly engage the $\mathrm{BH} 3$-binding groove of all anti-apoptotic proteins, others are more selective such as the BAD BH3 that binds BCL-2, BCL- $\mathrm{X}_{\mathrm{L}}$, and BCL-w and the NOXA BH3 that targets MCL-1 and BFL-1/A1 ${ }^{5}$. The differential binding capacity of BH3 domains and their mimetics is clinically relevant, as exemplified by the close relationship between inhibitor binding spectrum and biological activity. For example, ABT-737, the prototype small molecule $\mathrm{BH} 3$ mimetic modeled after the $\mathrm{BH} 3$ domain of $\mathrm{BAD}$, was designed to specifically target BCL-2 and BCL- $\mathrm{X}_{\mathrm{L}}$, and induces apoptosis in select cancers that are driven by these proteins ${ }^{9}$. However, ABT-737 fails to show efficacy against cancer cells that overexpress MCL-1, as this anti-apoptotic lies outside the molecule's range of binding activity ${ }^{12},{ }^{13}$. In an effort to overcome the challenge of designing precision small molecules to selectively target interaction surfaces that are comparatively large and more complex, we investigated whether nature's BH3 domains could provide a pharmacologic solution to anti-apoptotic specificity.

We chose MCL-1 as the template for this study because of its emerging role as a critical survival factor in a broad range of human cancers ${ }^{14}$. MCL-1 overexpression has been linked to the pathogenesis of a variety of refractory cancers, including multiple myeloma ${ }^{15}$, acute myeloid leukemia ${ }^{12}$, melanoma ${ }^{16}$, and poor prognosis breast cancer ${ }^{17}$. MCL-1 exerts its prosurvival activity at the mitochondrial outermembrane where it neutralizes pro-apoptotic proteins such as NOXA, PUMA, BIM, and BAK. The critical role of MCL-1 in apoptotic resistance has been highlighted by the sensitizing effects of small interfering RNAs that downregulate MCL-1 protein levels ${ }^{18}{ }^{20}$. Given the clear therapeutic rationale for targeting MCL-1, we sought to develop a selective MCL-1 inhibitor to elucidate the binding and specificity determinants, and interrogate its functional capacity to sensitize cancer cell apoptosis. 


\section{RESULTS}

\section{The MCL-1 BH3 helix is a selective inhibitor of MCL-1}

We previously applied hydrocarbon stapling to transform unfolded BID, BAD, and BIM

BH3 peptides into protease-resistant and cell-permeable a-helices that engage and modulate their intracellular targets for therapeutic benefit in preclinical models ${ }^{21}, 22$ and for mechanistic analyses ${ }^{23},{ }^{24}$. Here, we generated a library of Stabilized Alpha-Helix of BCL-2 domains (SAHBs) modeled after the BH3 domains of human BCL-2 family proteins in order to identify potent and selective inhibitors of MCL-1. We incorporated a pair of non-natural amino acids containing olefin tethers ${ }^{25}$ at the indicated $(i, i+4)$ positions of the noninteracting face of the $\mathrm{BH} 3$ helices (staple position "A"), followed by ruthenium-catalyzed olefin metathesis ${ }^{26}$, to yield a panel of hydrocarbon-stapled BH3 peptides (Fig. 1a, Supplementary Table 1). Fluorescence polarization assays (FPA) were performed to measure the binding affinity of fluorescently labeled SAHBs for recombinant human MCL-1 $\Delta \mathrm{N} \Delta \mathrm{C}$ (amino acids 172-320), a deletion construct that contains the BH3-binding pocket and affords enhanced expression, purity, and stability. SAHBs corresponding to the BH3 domains of (1) BH3-only proteins NOXA, PUMA, BID, and BIM, (2) multi-domain pro-apoptotic BAK, and (3) anti-apoptotic MCL-1 exhibited high affinity binding for MCL-1 ( $\left.\mathrm{K}_{D} \leq 50 \mathrm{nM}\right)$ (Fig. 1b). To identify MCL-1-selective SAHBs, we first screened for recombinant BCL- $\mathrm{X}_{\mathrm{L}} \Delta \mathrm{C}$ binding, which eliminated PUMA, BID, BIM, and BAK $\mathrm{SAHB}_{A} \mathrm{~S}$ (Supplementary Table 2a), and then for recombinant BFL1/A1 $\Delta$ C binding, which eliminated $\mathrm{NOXA} \mathrm{SAHB}_{A}$ (Supplementary Table 2b). Indeed, binding analysis of MCL-1 SAHB using an expanded panel of anti-apoptotic proteins, including MCL-1 $\Delta \mathrm{N} \Delta \mathrm{C}, \mathrm{BCL}-2 \Delta \mathrm{C}$, BCL- $\mathrm{X}_{\mathrm{L}} \Delta \mathrm{C}, \mathrm{BCL}-\mathrm{w} \Delta \mathrm{C}$ and BFL-1/A1 $\Delta \mathrm{C}$, confirmed that MCL-1 SAHB ${ }_{A}$ displayed potent and selective binding affinity for MCL-1 alone $\left(\mathrm{K}_{D}, 43 \mathrm{nM}\right)$ (Fig. 1c).

\section{Binding and specificity determinants of the MCL-1 BH3 domain}

To define the binding and specificity determinants for the interaction between the MCL-1 BH3 helix and MCL- $1 \Delta \mathrm{N} \Delta \mathrm{C}$, we performed alanine scanning, site-directed mutagenesis, and staple scanning. Amino acid residues within MCL-1 SAHB $A$ were sequentially replaced with alanine and the corresponding fluorescently labeled SAHBs were tested for MCL- $1 \Delta \mathrm{N} \Delta \mathrm{C}$ binding by FPA. The alanine scan was supplemented with glutamate mutagenesis of alanine and glycine residues. Whereas mutagenesis of $\mathrm{N}$ - and $\mathrm{C}$ - terminal residues had little to no impact on MCL- $1 \Delta \mathrm{N} \Delta \mathrm{C}$ binding affinity, alanine mutagenesis of L213, R214, V216, G217, D218 and V220 decreased the binding affinity of MCL-1 SAHB $A$ for MCL-1 $\Delta \mathrm{N} \Delta \mathrm{C}$ by 10- to 100-fold, revealing the key MCL-1 BH3 residues for MCL- $1 \Delta \mathrm{N} \Delta \mathrm{C}$ engagement (Fig. 2a). Comparative analysis of BH3 domain sequences indicated that the combination of core hydrophobic residues L213, V216, and V220 is unique to MCL-1 BH3 (Fig. 1a) and alanine mutagenesis of any one of these hydrophobic residues is especially detrimental to MCL- $1 \Delta \mathrm{N} \Delta \mathrm{C}$ binding. Interestingly, BAD BH3, which exhibits a restricted binding profile to BCL-2, BCL- $\mathrm{X}_{\mathrm{L}}$, and BCL-w, and BIM BH3, which broadly engages anti-apoptotic proteins, possess a phenylalanine at the position corresponding to V220 in MCL-1 BH3 (Supplementary Fig. 1). Scanning mutagenesis of the BIM BH3 sequence previously documented that replacement of this phenylalanine with alanine, glutamate, or lysine abrogated BCL- $\mathrm{X}_{\mathrm{L}}$ binding but had minimal impact on MCL-1 
binding ${ }^{27},{ }^{28}$. We find that a single V220F point mutation in $\mathrm{MCL}-1 \mathrm{SAHB}_{A}$ abolished selectivity for MCL-1 $\Delta \mathrm{N} \Delta \mathrm{C}$, conferring binding activity to both MCL-1 $\Delta \mathrm{N} \Delta \mathrm{C}\left(\mathrm{K}_{D}, 191\right.$ $\mathrm{nM})$ and BCL- $\mathrm{X}_{\mathrm{L}} \Delta \mathrm{C}\left(\mathrm{K}_{D}, 89 \mathrm{nM}\right)$ (Fig. $\left.2 \mathrm{~b}\right)$. Whereas certain binding determinants such as the conserved amino acids L213, R214, G217, and D218 are shared among many BH3 domains, other discrete residues in the appropriate context, such as V220 in MCL-1 BH3, can dictate selectivity.

We next performed a "staple scan" that effectively replaced pairs of amino acid residues within the BH3 sequence with crosslinked norleucine-like side chains to (1) address which surface along the MCL-1 BH3 helix is essential to MCL-1 $\Delta \mathrm{N} \Delta \mathrm{C}$ engagement and (2) sample alternate staple positions to identify constructs with optimal a-helicity and binding activity for biological studies. In agreement with the alanine scan, mutagenesis of residues E211, R215, G219, Q221, N223, E225, and A227, and insertion of staples at $i, i+4$ pairings of these sites, did not disrupt the MCL-1 $\Delta \mathrm{N} \Delta \mathrm{C}$ interaction (Fig. 2c). However, placement of the crosslink at positions G217 to Q221 abrogated binding activity, consistent with disruption of the critical hydrophobic interface between MCL-1 SAHB $C$ and MCL-1 $\Delta \mathrm{N} \Delta \mathrm{C}$ by the hydrocarbon staple. Among the MCL-1 SAHBs generated, MCL-1 SAHB ${ }_{D}$ exhibited high a-helical content $(\sim 90 \%)$ and the strongest binding activity $\left(\mathrm{K}_{D}, 10 \mathrm{nM}\right)$, achieving 4fold enhancement in MCL-1 $\Delta \mathrm{N} \Delta \mathrm{C}$ affinity compared to the parental MCL-1 $\mathrm{SAHB}_{A}$ while retaining MCL-1 $\Delta \mathrm{N} \Delta \mathrm{C}$ selectivity (Fig. 2c, Supplementary Fig. 2, 3).

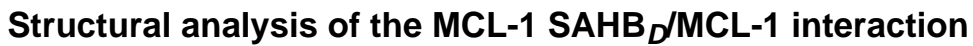

To structurally define the interactions between a selective MCL-1 ligand and its target, we determined the crystal structure of our strongest interactor, MCL-1 SAHB ${ }_{D}$, in complex with MCL-1 $\Delta \mathrm{N} \Delta \mathrm{C}$ at 2.32- $\AA$ resolution (Fig. 3, Supplementary Table 3, PDB 3MK8). Analysis of the three-dimensional structure revealed that MCL-1 $\mathrm{SAHB}_{D}$ is an a-helix that engages MCL- $1 \Delta \mathrm{N} \Delta \mathrm{C}$ at the canonical BH3-binding groove comprised of helices a2 (BH3) and portions of a3, a4, a5 (BH1), and a8 (BH2) (Fig. 3a). Hydrophobic residues L213, V216, G217, and V220 of MCL-1 SAHB ${ }_{D}$ make direct contact with the hydrophobic groove at the surface of MCL-1 $\Delta \mathrm{N} \Delta \mathrm{C}$ (Fig. 3b), consistent with the negative ramifications of alanine mutagenesis of these amino acids in MCL-1 $\mathrm{SAHB}_{A}$ (Fig. 2a). The hydrophobic interactions are reinforced by a salt bridge between MCL-1 SAHB ${ }_{D}$ D218 and MCL-1 $\triangle \mathrm{N} \triangle \mathrm{C}$ R263; these residues also participate in a hydrogen bond cluster that includes MCL-1 $\Delta \mathrm{N} \Delta \mathrm{C}$ D256 and N260 (Fig. 3a). Adjacent to this cluster, MCL-1 SAHB ${ }_{D}$ R214 lies in close proximity to MCL-1 $\triangle \mathrm{N} \Delta \mathrm{C}$ S255 and D256, which forms the edge of an intricate polar network that complements the binding surface of MCL-1 SAHB $D$ N-terminal to the staple site.

The differential binding activities of MCL-1 SAHBs $A-E$ are consistent with the structure of the MCL-1 SAHB ${ }_{D} / \mathrm{MCL}-1 \Delta \mathrm{N} \Delta \mathrm{C}$ complex. MCL-1 SAHB $C$ is the only construct that exhibits poor binding activity and, based on the structure, it bears the only staple location (G217,Q221) that would sterically clash with the binding surface. Interestingly, the hydrocarbon staple of MCL-1 $\mathrm{SAHB}_{D}$, whose alkene functionality is in the cis conformation, makes discrete hydrophobic contacts with the perimeter of the MCL-1 $\Delta \mathrm{N} \Delta \mathrm{C}$ binding site. A methyl group of the a,a-dimethyl functionality occupies a groove consisting 
of MCL-1 $\Delta \mathrm{N} \Delta \mathrm{C} \mathrm{G} 262, \mathrm{~F} 318$, and F319, and additional contacts are also evident for the aliphatic side chain (Fig. 3c). Thus, the superior binding affinity of MCL-1 SAHB ${ }_{D}$ may derive both from its enhanced a-helicity (Supplementary Fig. 2) and the recruitment of additional hydrophobic contacts by the staple itself. Indeed, these structural data highlight the potential to harness the staple functionality to optimize the potency of SAHB ligands while retaining their natural biological specificities.

\section{MCL-1 SAHB ${ }_{D}$ targets MCL-1 and sensitizes apoptosis}

We next conducted a series of functional studies to determine if MCL-1 SAHB $D$ could effectively target MCL-1 and sensitize mitochondrial apoptosis in vitro and in cells. We first performed a competitive FPA to measure the capacity of MCL-1 SAHBs to dissociate a BAK BH3 helix from MCL-1 $\Delta \mathrm{N} \Delta \mathrm{C}$, simulating the displacement activity required for in situ function. Consistent with the direct binding data (Fig. 2c), MCL-1 SAHB ${ }_{D}$ was most effective at antagonizing the interaction between FITC-BAK SAHB ${ }_{A}$ and MCL-1 $\Delta \mathrm{N} \Delta \mathrm{C}$ (Fig. 4a). We next conducted mitochondrial assays to determine if the ability of MCL-1 $\mathrm{SAHB}_{D}$ to disrupt the FITC-BAK SAHB $/ \mathrm{MCL}-1 \Delta \mathrm{N} \Delta \mathrm{C}$ complex translated into SAHBmediated sensitization of BAK-induced cytochrome $c$ release. Wild-type mouse liver mitochondria that contain $\mathrm{BAK}$ were exposed to $\mathrm{BID} \mathrm{BH} 3$, a direct activator of $\mathrm{BAK}^{29}$, in the presence and absence of a serial dilution of MCL-1 SAHB ${ }_{D}$. Whereas MCL-1 SAHB $D$ had no effect on the mitochondria in the absence of BID BH3, addition of MCL-1 SAHB $D$ to $\mathrm{BID} \mathrm{BH} 3$-exposed mitochondria triggered dose-responsive enhancement of BAKmediated cytochrome $c$ release (Fig. 4b). To confirm that cytochrome $c$ release specifically derived from BAK activation, the identical experiment was performed with $\mathrm{Bak}^{-/-}$ mitochondria, and no release was observed (Fig. 4b).

To extend these findings to a cellular context, we first confirmed that MCL-1 SAHB ${ }_{D}$ could target cellular MCL-1 and dissociate native MCL-1/BAK complexes. We synthesized a photoreactive SAHB (pSAHB) that replaced L210 with a non-natural amino acid bearing a benzophenone moiety (4-benzoylphenylalanine, Bpa), which covalently crosslinks to protein targets upon exposure to ultraviolet (UV) light $^{30}$. We incubated N-terminal biotinylated adducts of MCL-1 pSAHB ${ }_{D}$ or MCL-1 $\mathrm{SAHB}_{D}$ with cellular extract from OPM2 multiple myeloma cells in the presence of UV irradiation, followed by streptavidin-based affinity purification, stringent washes to remove non-covalent binders, elution, electrophoresis, and MCL-1 western analysis. Indeed, the photoreactive analog of MCL-1 SAHB ${ }_{D}$ effectively trapped native MCL-1 contained within the cellular lysate, whereas the negative control SAHB that lacked the benzophenone moiety showed no covalent capture-based isolation of MCL-1 (Fig. 4c, Supplementary Fig. 4a). Cultured OPM2 cells were then treated with vehicle or increasing concentrations of MCL-1 SAHB ${ }_{D}$, followed by immunoprecipitation of MCL-1. BAK western analysis revealed co-immunoprecipitation of MCL-1/BAK from vehicle-treated cells but dose-responsive dissociation of the MCL-1/BAK interaction by MCL-1 SAHB ${ }_{D}$ (Fig. 4d, Supplementary Fig. 4b). Taken together, these mechanistic data demonstrate that MCL-1 $\mathrm{SAHB}_{D}$ can directly target native MCL-1 in a complex protein mixture, disrupt the inhibitory MCL-1/BAK interaction in vitro and in cells, and sensitize BAK-mediated mitochondrial cytochrome $c$ release. 
Importantly, selective liberation of pro-apoptotic proteins from MCL-1 may not activate cellular apoptosis if alternative anti-apoptotics are present at sufficient levels to bind and neutralize them. From a functional standpoint, a selective MCL-1 inhibitor would instead be expected to promote apoptosis in cells that employ MCL-1 as a key component of the survival response to a particular stress stimulus. Thus, to examine the functional consequences of selective pharmacologic blockade of MCL-1 in cells, we tested the capacity of MCL-1 $\mathrm{SAHB}_{D}$ to sensitize cancer cells to death receptor agonists, whose activity is blunted by MCL-1 and enhanced by MCL-1 knockdown ${ }^{19},{ }^{20}, 31,32$. Jurkat T-cell leukemia and OPM2 cells were first exposed to serial dilutions of MCL-1 SAHB $D$ and the extrinsic pathway activators TRAIL and Fas ligand (FasL) as single agents to obtain baseline viability measurements (Fig. 5a,b, Supplementary Fig. 5). MCL-1 SAHB ${ }_{D}$ had no effect on cell viability even at $40 \mu \mathrm{M}$ dosing. Jurkat cells exhibited dose-responsive cytotoxicity in response to both TRAIL and FasL, whereas OPM2 cells were sensitive to TRAIL but not FasL (Supplementary Fig. 5). To determine if direct and selective MCL-1 blockade could sensitize the cells to TRAIL- and FasL-induced apoptosis, a serial dilution of MCL-1 $\mathrm{SAHB}_{D}$ was combined with low-dose death receptor ligands. MCL-1 SAHB ${ }_{D}$ doseresponsively sensitized Jurkat cells to both TRAIL and FasL (Fig. 5a), and selectively sensitized OPM2 cells to TRAIL (Fig. 5b). MCL-1 SAHB ${ }_{D}$ had no effect on OPM2 cells exposed to FasL, consistent with the lack of response of OPM2 cells to FasL treatment (Supplementary Fig. 5). To confirm that MCL-1 $\mathrm{SAHB}_{D}$-induced sensitization was caspasedependent, cell viability testing was also conducted in the presence of the pan-caspase inhibitor, z-VAD, which completely abrogated the negative effects on cell viability (Fig. 5a,b). Consistent with these data, MCL-1 SAHB ${ }_{D}$ triggered dose-responsive caspase 3/7 activation when used in combination with low dose TRAIL and FasL in Jurkat cells (Fig. 5c) and with TRAIL, but not FasL, in OPM2 cells (Fig. 5d). Importantly, BFL-1/A1 $\mathrm{SAHB}_{A}$, which displayed no binding activity toward anti-apoptotic proteins, did not sensitize Jurkat cells to TRAIL or FasL (Supplementary Fig. 6a). As a positive control, we sought to generate an additional MCL-1-selective SAHB with a different BH3 sequence. A limited staple scan revealed that relocalizing the staple from R31,K35 in NOXA $\mathrm{SAHB}_{A}$ to the $\mathrm{A} 26, \mathrm{R} 30$ position in NOXA $\mathrm{SAHB}_{B}$ narrowed the natural MCL-1 and BFL-1/A1 binding selectivity to MCL-1 only, and $\mathrm{NOXA} \mathrm{SAHB}_{B}$ indeed functioned like MCL-1 $\mathrm{SAHB}_{D}$ in this sensitization study (Supplementary Fig. 6b). Whereas MCL-1 SAHB ${ }_{D}$ and $\mathrm{NOXA} \mathrm{SAHB}_{B}$ showed similar levels of cellular uptake, the negative control BFL-1/A1 $\mathrm{SAHB}_{A}$ exhibited even higher intracellular levels, confirming that the inactivity of BFL-1/A1 $\mathrm{SAHB}_{A}$ did not derive from a lack of cell penetrance (Supplementary Fig. 6c). Taken together, our mechanistic and functional data demonstrate that MCL-1 $\mathrm{SAHB}_{D}$ is a selective, cell-permeable MCL-1 antagonist, which sensitizes cancer cells to apoptotic stimuli that are suppressed by MCL-1.

\section{DISCUSSION}

BCL-2 proteins, like many protein families, are comprised of numerous members that share a high percentage of sequence identity and functional homology. It is the differences among these homologous proteins, however, that give rise to their unique interactions and spectra of activity. When implicated in pathologic protein interactions, it may be desirable to neutralize 
all anti-apoptotic family members or a discrete subset, with the drug profile of choice dictated by the nature and severity of the disease. In the case of targeting anti-apoptotic BCL-2 family proteins that cause uncontrolled cell survival, an ideal pharmacologic toolbox would contain agents that target individual, subsets, and all members. Achieving this goal requires careful structural dissection of both the unique and common elements of $\mathrm{BH} 3$ interactions with anti-apoptotic targets.

Anti-apoptotic MCL-1 is a high priority target for developmental cancer therapeutics due to its emergence as a formidable and pervasive oncogenic protein ${ }^{14}$ and chemoresistance factor ${ }^{33},{ }^{34}$. Guided by the natural binding selectivities of $\mathrm{BH} 3$ helices for discrete antiapoptotic proteins, we have identified a potent and exclusive inhibitor of MCL-1 based on structural reinforcement of its own a-helical BH3 domain. Indeed, our findings reveal the structural basis for a previous observation that MCL-1 short (MCL-1S), a splice variant of MCL-1 that lacks BH1, BH2, and C-terminal transmembrane domains but retains the $\mathrm{BH}$, only interacted with MCL-1 in a yeast two hybrid analysis of multidomain pro- and antiapoptotic BCL-2 family proteins ${ }^{35}$. The potent and selective interaction between the MCL-1 BH3 helix and MCL-1 is mediated by highly conserved BH3 elements, such as L213, R214, G217, and D218, and a uniquely branched hydrophobic interface comprised of L213, Val216, and V220. A single V220F mutation eliminates the MCL-1 binding exclusivity of MCL-1 SAHB ${ }_{A}$, underscoring the importance of individual amino acid contacts in dictating the binding spectrum of $\mathrm{BH} 3$ a-helices. Compared to the structures of other $\mathrm{BH} 3$ peptides in complex with MCL-1 (e.g. BID [2KBW], BIM [2PQK, 2NL9], NOXA [2ROD, 2NLA], PUMA [2ROC]), MCL-1 $\mathrm{SAHB}_{D}$ has a shorter structured core, which is confined to approximately three $a$-helical turns. The critical contacts that lie within this a-helical unit drive the potency and selectivity of the interaction, as demonstrated by site directed mutagenesis of MCL-1 SAHB ${ }_{A}$. Interestingly, the "D" staple replaces the polar Q221 residue of MCL-1 BH3 and makes hydrophobic contact with G262 Ca (a5) and F318 (a8) of MCL-1, mirroring the binding of a similarly oriented Y35 residue in murine NOXA-A BH3 with G243 (a5) and F299 (a8) of murine MCL-1 (2ROD). Thus, by engaging in additional hydrophobic contacts at the perimeter of the core binding interface, the hydrocarbon staple may directly contribute to the enhanced affinity of MCL-1 SAHB ${ }_{D}$ compared to its differentially stapled analogs without compromising specificity.

From a functional standpoint, MCL-1 $\mathrm{SAHB}_{D}$ effectively targets native MCL-1, disrupts its capacity to suppress the death pathway through protein interaction, and sensitizes caspasedependent cancer cell apoptosis in the context of death receptor stimulation. Whereas TRAIL ligand and antibody-based TRAIL receptor agonists are currently being evaluated in clinical trials, they can be rendered ineffective by MCL-1 expression ${ }^{36}, 37$, underscoring the clinical relevance of our findings. By identifying the critical binding and specificity determinants for selective MCL-1 inhibition by a natural BH3 domain, our data provide a blueprint for the development of novel therapeutics to reactivate apoptosis in diseases driven by pathologic MCL-1-mediated cell survival and chemoresistance. 


\section{METHODS}

\section{Peptide synthesis}

SAHBs were synthesized, purified, and characterized by circular dichroism as described in detail $^{38}$. All peptides were purified by liquid chromatography-mass spectroscopy to $>95 \%$ purity and quantitated by amino acid analysis. For CD analysis, MCL-1 SAHBs were dissolved in a $5 \mathrm{mM}$ potassium phosphate solution $(\mathrm{pH} 7.5)$ to achieve a target concentration of $20 \mu \mathrm{M}$. For all other experiments, MCL-1 SAHBs were reconstituted in deionized water. The compositions of human SAHBs used in this study are listed in Supplementary Table 1.

\section{Anti-apoptotic protein production}

Recombinant and tagless MCL-1 $\Delta \mathrm{N} \Delta \mathrm{C}, \mathrm{BCL}-2 \Delta \mathrm{C}, \mathrm{BCL}-\mathrm{X}_{\mathrm{L}} \Delta \mathrm{C}, \mathrm{BCL}-\mathrm{w} \Delta \mathrm{C}$, and BFL1/ $\mathrm{A} 1 \Delta \mathrm{C}$ were expressed and purified as previously reported ${ }^{39}$ and as described in the Supplementary Methods.

\section{Fluorescence polarization binding assays}

Binding assays were performed as previously described ${ }^{39}$. FITC-SAHB $(50 \mathrm{nM})$ was added to serial dilutions of recombinant protein in binding buffer $(50 \mathrm{mM}$ Tris, $100 \mathrm{mM} \mathrm{NaCl}, \mathrm{pH}$ 8.0). For competition assays, serial dilutions of acetylated MCL-1 SAHBs were mixed with FITC-BAK SAHB $(25 \mathrm{nM})$, followed by addition of MCL-1 $\Delta \mathrm{N} \Delta \mathrm{C}(100 \mathrm{nM})$ diluted in binding buffer. Multiwell plates were incubated in the dark at room temperature until equilibrium was reached and fluorescence polarization (mP units) measured by microplate reader (SpectraMax, Molecular Devices). For direct binding experiments, dissociation constants $\left(\mathrm{K}_{D}\right)$ were calculated by nonlinear regression analysis of dose-response curves using Prism software (Graphpad), as described ${ }^{39}$. For competition experiments, $\mathrm{K}_{i}$ values were determined by nonlinear regression analysis of dose-response curves using a one-site competition model.

\section{X-ray crystallography}

MCL- $1 \Delta \mathrm{N} \Delta \mathrm{C}$ protein $(6.3 \mathrm{mg} / \mathrm{mL})$ in $50 \mathrm{mM} \mathrm{NaCl}, 20 \mathrm{mM}$ Tris, $2 \mathrm{mM}$ DTT, pH 7.4 was incubated with an equimolar ratio of MCL-1 $\mathrm{SAHB}_{D}$ reconstituted in water. The mixture (1 $\mu \mathrm{L}$ ) was added to an equal volume of the reservoir solution (0.1 M Bis Tris, 28\% PEG MME 2000, pH 6.5), and crystals were grown by vapor diffusion using the sitting drop method. Crystals were flash frozen in liquid nitrogen and x-ray diffraction data for the P212121 crystal were collected at the Argonne National Laboratory using the advanced photon beamline 24-ID-C, and scaled to 2.32- $\AA$. HLK2000 ${ }^{40}$ was used for data processing and phases were obtained by molecular replacement of chain A of PDB 3KJ0 (MCL-1 chain) using PHASER ${ }^{41}$. Iterative rounds of refinement using TLS and model building were performed using PHENIX ${ }^{42}$ and COOT $^{43}$, respectively (PDB 3MK8; $R_{\text {work }} / R_{\text {free, }}$, 23.1/27.5). Topology and parameter files were created for non-natural amino acid residues using published bond lengths and angles ${ }^{44}$. MolProbity ${ }^{45}$ was used to validate the structure (94.74\% Ramachandran favored, $0.66 \%$ Ramachandran outliers, 0 bad rotamers). 


\section{Cytochrome $c$ release assays}

Mouse liver mitochondria $(0.5 \mathrm{mg} / \mathrm{mL})$ were isolated and cytochrome $c$ release assays performed according to established methods ${ }^{39}$ and as described in the Supplementary Methods.

\section{MCL-1 SAHB photocrosslinking}

MCL-1 SAHB photocrosslinking was performed using MCL-1 pSAHB ${ }_{D}(10 \mu \mathrm{M})$, OPM2 cellular lysates, and $365 \mathrm{~nm}$ ultraviolet light as described in detail in the Supplementary Methods.

\section{MCL-1 immunoprecipitation assay}

OPM2 cells $\left(1 \times 10^{7}\right)$ were incubated with vehicle or MCL- $1 \mathrm{SAHB}_{D}$ at the indicated concentrations in Opti-MEM medium (Invitrogen) at $37^{\circ} \mathrm{C}$ for 4 hours. Cells were washed once with cold PBS and lysed on ice with $500 \mu \mathrm{L}$ of cold NP-40 lysis buffer (50 mM Tris pH 7.4, $150 \mathrm{mM} \mathrm{NaCl}, 1 \mathrm{mM}$ EDTA, $1 \mathrm{mM}$ DTT, $0.5 \%$ NP40, complete protease inhibitor pellet). Cellular debris was pelleted at $14,000 \times g$ for 10 minutes at $4^{\circ} \mathrm{C}$ and the supernatant was collected and exposed to pre-equilibrated protein A/G sepharose beads. The pre-cleared supernatant was incubated with anti-MCL-1 antibody (S-19, Santa Cruz Biotechnology) overnight at $4^{\circ} \mathrm{C}$, followed by the addition of protein $\mathrm{A} / \mathrm{G}$ sepharose beads for 1 hour. The beads were then pelleted, washed with NP-40 lysis buffer for 10 minutes at $4^{\circ} \mathrm{C}$ three times, and the protein sample eluted from the beads by heating at $90^{\circ} \mathrm{C}$ for 10 minutes in SDS loading buffer. The immunoprecipitates were subjected to electrophoresis and western analysis using the BAK(NT) antibody (CalBiochem).

\section{Cell viability assay}

OPM2 multiple myeloma and Jurkat T-cell leukemia cells were maintained in RPMI 1640 medium (Invitrogen) supplemented with $10 \%$ fetal bovine serum, $100 \mathrm{U} / \mathrm{mL}$ penicillin, 100 $\mu \mathrm{g} / \mathrm{mL}$ streptomycin, $2 \mathrm{mM}$ L-glutamine, $50 \mathrm{mM}$ HEPES and $50 \mu \mathrm{M} \beta$-mercaptoethanol. For viability testing, OPM2 and Jurkat cells $\left(4 \times 10^{4}\right)$ were treated with the indicated agents in Opti-MEM media at $37^{\circ} \mathrm{C}$ in a final volume of $100 \mu \mathrm{L}$. Cell viability was measured at 24 hours by MTT assay (Sigma). For synergy studies with TRAIL or FasL, cells were treated simultaneously with MCL-1 SAHB ${ }_{D}$ and the death receptor ligands in the presence or absence of the pan-caspase inhibitor z-VAD $(50 \mu \mathrm{M})$, which was administered to cells 30 minutes prior to treatment with the pro-apoptotic agents.

\section{Capsase 3/7 activation assay}

OPM2 and Jurkat cells $\left(2 \times 10^{4}\right.$ cells $)$ were treated with the indicated agents in Opti-MEM media at $37^{\circ} \mathrm{C}$ in a final volume of $50 \mu \mathrm{L}$. Caspase $3 / 7$ activation was measured at 4 hours using the ApoONE Caspase 3/7 kit (Promega). For synergy studies with TRAIL or FasL, cells were treated simultaneously with MCL-1 $\mathrm{SAHB}_{D}$ and the death receptor ligands.

\section{Supplementary Material}

Refer to Web version on PubMed Central for supplementary material. 


\section{Acknowledgments}

We thank E. Smith for editorial and graphics support, R.A. Grant for input on the crystallography experiments, xray data collection and structural analysis, and C.H. Yun and M.J. Eck for assistance in generating the hydrocarbon staple parameter file used in the structural refinement. This work was supported by NIH grant 5P01CA92625 and a Burroughs Wellcome Career Award in the Biomedical Sciences to L.D.W., a Ruth L. Kirschstein National Research Service Award 1F31CA144566 to M.L.S., and NIH award 5RO1GM084181 to A.E.K. X-ray diffraction data were acquired at the Advanced Photon Source on the Northeastern Collaborative Access Team beamlines, which are supported by award RR-15301 from the National Center for Research Resources at the NIH. Use of the Advanced Photon Source is supported by the U.S. Department of Energy, Office of Basic Energy Sciences, under Contract No. DE-AC02-06CH11357.

\section{References}

1. Tsujimoto Y, Cossman J, Jaffe E, Croce CM. Involvement of the bcl-2 gene in human follicular lymphoma. Science. 1985; 228:1440-3. [PubMed: 3874430]

2. Danial NN, Korsmeyer SJ. Cell death: critical control points. Cell. 2004; 116:205-19. [PubMed: 14744432]

3. Sattler M, et al. Structure of Bcl-xL-Bak peptide complex: recognition between regulators of apoptosis. Science. 1997; 275:983-6. [PubMed: 9020082]

4. Muchmore SW, et al. X-ray and NMR structure of human Bcl-xL, an inhibitor of programmed cell death. Nature. 1996; 381:335-41. [PubMed: 8692274]

5. Chen $\mathrm{L}$, et al. Differential targeting of prosurvival Bcl-2 proteins by their $\mathrm{BH} 3$-only ligands allows complementary apoptotic function. Mol Cell. 2005; 17:393-403. [PubMed: 15694340]

6. Zhai D, Jin C, Huang Z, Satterthwait AC, Reed JC. Differential regulation of Bax and Bak by antiapoptotic Bcl-2 family proteins Bcl-B and Mcl-1. J Biol Chem. 2008; 283:9580-6. [PubMed: 18178565]

7. Kitada S, et al. Discovery, characterization, and structure-activity relationships studies of proapoptotic polyphenols targeting B-cell lymphocyte/leukemia-2 proteins. J Med Chem. 2003; 46:4259-64. [PubMed: 13678404]

8. Nguyen M, et al. Small molecule obatoclax (GX15-070) antagonizes MCL-1 and overcomes MCL-1-mediated resistance to apoptosis. Proc Natl Acad Sci U S A. 2007; 104:19512-7. [PubMed: 18040043]

9. Oltersdorf T, et al. An inhibitor of Bcl-2 family proteins induces regression of solid tumours. Nature. 2005; 435:677-81. [PubMed: 15902208]

10. Tse C, et al. ABT-263: a potent and orally bioavailable Bcl-2 family inhibitor. Cancer Res. 2008; 68:3421-8. [PubMed: 18451170]

11. Wang G, et al. Structure-based design of potent small-molecule inhibitors of anti-apoptotic Bcl-2 proteins. J Med Chem. 2006; 49:6139-42. [PubMed: 17034116]

12. Konopleva M, et al. Mechanisms of apoptosis sensitivity and resistance to the $\mathrm{BH} 3$ mimetic ABT-737 in acute myeloid leukemia. Cancer Cell. 2006; 10:375-88. [PubMed: 17097560]

13. van Delft MF, et al. The BH3 mimetic ABT-737 targets selective Bcl-2 proteins and efficiently induces apoptosis via Bak/Bax if Mcl-1 is neutralized. Cancer Cell. 2006; 10:389-99. [PubMed: 17097561]

14. Beroukhim R, et al. The landscape of somatic copy-number alteration across human cancers. Nature. 2010; 463:899-905. [PubMed: 20164920]

15. Zhang B, Gojo I, Fenton RG. Myeloid cell factor-1 is a critical survival factor for multiple myeloma. Blood. 2002; 99:1885-93. [PubMed: 11877256]

16. Boisvert-Adamo K, Longmate W, Abel EV, Aplin AE. Mcl-1 is required for melanoma cell resistance to anoikis. Mol Cancer Res. 2009; 7:549-56. [PubMed: 19372583]

17. Ding Q, et al. Myeloid Cell Leukemia-1 Inversely Correlates with Glycogen Synthase Kinase-3 3 beta\} Activity and Associates with Poor Prognosis in Human Breast Cancer. Cancer Res. 2007; 67:4564-71. [PubMed: 17495324] 
18. Lin X, et al. 'Seed' analysis of off-target siRNAs reveals an essential role of Mcl-1 in resistance to the small-molecule Bcl-2/Bcl-XL inhibitor ABT-737. Oncogene. 2007; 26:3972-9. [PubMed: 17173063]

19. Meng XW, et al. Mcl-1 as a buffer for proapoptotic Bcl-2 family members during TRAIL-induced apoptosis: a mechanistic basis for sorafenib (Bay 43-9006)-induced TRAIL sensitization. J Biol Chem. 2007; 282:29831-46. [PubMed: 17698840]

20. Taniai M, et al. Mcl-1 mediates tumor necrosis factor-related apoptosis-inducing ligand resistance in human cholangiocarcinoma cells. Cancer Res. 2004; 64:3517-24. [PubMed: 15150106]

21. Danial NN, et al. Dual role of proapoptotic BAD in insulin secretion and beta cell survival. Nat Med. 2008; 14:144-53. [PubMed: 18223655]

22. Walensky LD, et al. Activation of apoptosis in vivo by a hydrocarbon-stapled BH3 helix. Science. 2004; 305:1466-70. [PubMed: 15353804]

23. Gavathiotis E, et al. BAX activation is initiated at a novel interaction site. Nature. 2008; 455:107681. [PubMed: 18948948]

24. Walensky LD, et al. A stapled BID BH3 helix directly binds and activates BAX. Mol Cell. 2006; 24:199-210. [PubMed: 17052454]

25. Schafmeister C, Po J, Verdine G. An all-hydrocarbon cross-linking system for enhancing the helicity and metabolic stability of peptides. J Am Chem Soc. 2000; 122:5891-5892.

26. Blackwell HE, et al. Ring-Closing Metathesis of Olefinic Peptides: Design, Synthesis, and Structural Characterization of Macrocyclic Helical Peptides. J Org Chem. 2001; 66:5291-5302. [PubMed: 11485448]

27. Boersma MD, Sadowsky JD, Tomita YA, Gellman SH. Hydrophile scanning as a complement to alanine scanning for exploring and manipulating protein-protein recognition: application to the Bim BH3 domain. Protein Sci. 2008; 17:1232-40. [PubMed: 18467496]

28. Day CL, et al. Structure of the BH3 domains from the p53-inducible BH3-only proteins Noxa and Puma in complex with Mcl-1. J Mol Biol. 2008; 380:958-71. [PubMed: 18589438]

29. Letai A, et al. Distinct BH3 domains either sensitize or activate mitochondrial apoptosis, serving as prototype cancer therapeutics. Cancer Cell. 2002; 2:183-192. [PubMed: 12242151]

30. Saghatelian A, Jessani N, Joseph A, Humphrey M, Cravatt BF. Activity-based probes for the proteomic profiling of metalloproteases. Proc Natl Acad Sci U S A. 2004; 101:10000-5. [PubMed: 15220480]

31. Clohessy JG, Zhuang J, de Boer J, Gil-Gomez G, Brady HJ. Mcl-1 interacts with truncated Bid and inhibits its induction of cytochrome $\mathrm{c}$ release and its role in receptor-mediated apoptosis. $\mathrm{J}$ Biol Chem. 2006; 281:5750-9. [PubMed: 16380381]

32. Han J, Goldstein LA, Gastman BR, Rabinowich H. Interrelated roles for Mcl-1 and BIM in regulation of TRAIL-mediated mitochondrial apoptosis. J Biol Chem. 2006; 281:10153-63. [PubMed: 16478725]

33. Akgul C. Mcl-1 is a potential therapeutic target in multiple types of cancer. Cell Mol Life Sci. 2009; 66:1326-36. [PubMed: 19099185]

34. Warr MR, Shore GC. Unique biology of Mcl-1: therapeutic opportunities in cancer. Curr Mol Med. 2008; 8:138-47. [PubMed: 18336294]

35. Bae J, Leo CP, Hsu SY, Hsueh AJ. MCL-1S, a splicing variant of the antiapoptotic BCL-2 family member MCL-1, encodes a proapoptotic protein possessing only the BH3 domain. J Biol Chem. 2000; 275:25255-61. [PubMed: 10837489]

36. Kim SH, Ricci MS, El-Deiry WS. Mcl-1: a gateway to TRAIL sensitization. Cancer Res. 2008; 68:2062-4. [PubMed: 18381408]

37. Ricci MS, et al. Reduction of TRAIL-induced Mcl-1 and cIAP2 by c-Myc or sorafenib sensitizes resistant human cancer cells to TRAIL-induced death. Cancer Cell. 2007; 12:66-80. [PubMed: 17613437]

38. Bird GH, Bernal F, Pitter K, Walensky LD. Chapter 22 Synthesis and biophysical characterization of Stabilized Alpha-helices of BCL-2 Domains. Methods Enzymol. 2008; 446:369-86. [PubMed: 18603134] 
39. Pitter K, Bernal F, LaBelle JL, Walensky LD. Chapter 23 Dissection of the BCL-2 family signaling network with Stabilized Alpha-Helices of BCL-2 Domains. Methods Enzymol. 2008; 446:387-408. [PubMed: 18603135]

40. Otwinowski Z, Minor W. Processing of X-ray diffraction data collected in oscillation mode. Methods Enzymol. 1997; 276:307-326.

41. Storoni LC, McCoy AJ, Read RJ. Likelihood-enhanced fast rotation functions. Acta Crystallogr D Biol Crystallogr. 2004; 60:432-8. [PubMed: 14993666]

42. Adams PD, et al. PHENIX: building new software for automated crystallographic structure determination. Acta Crystallogr D Biol Crystallogr. 2002; 58:1948-54. [PubMed: 12393927]

43. Emsley P, Cowtan K. Coot: model-building tools for molecular graphics. Acta Crystallogr D Biol Crystallogr. 2004; 60:2126-32. [PubMed: 15572765]

44. Lynch VM, Tanaka T, Fishpaugh JR, Martin SF, Davis BE. Structure of (+/-)-(1R*,4S*,6S*)-1benzyloxy-4,8,11,11-tetramethyl-6-phenylthio-bicyclo[5.3.1]undec-7-en-3-one. Acta Crystallogr C. 1990; 46 ( Pt 7):1351-3. [PubMed: 2222933]

45. Davis IW, et al. MolProbity: all-atom contacts and structure validation for proteins and nucleic acids. Nucleic Acids Res. 2007; 35:W375-83. [PubMed: 17452350] 

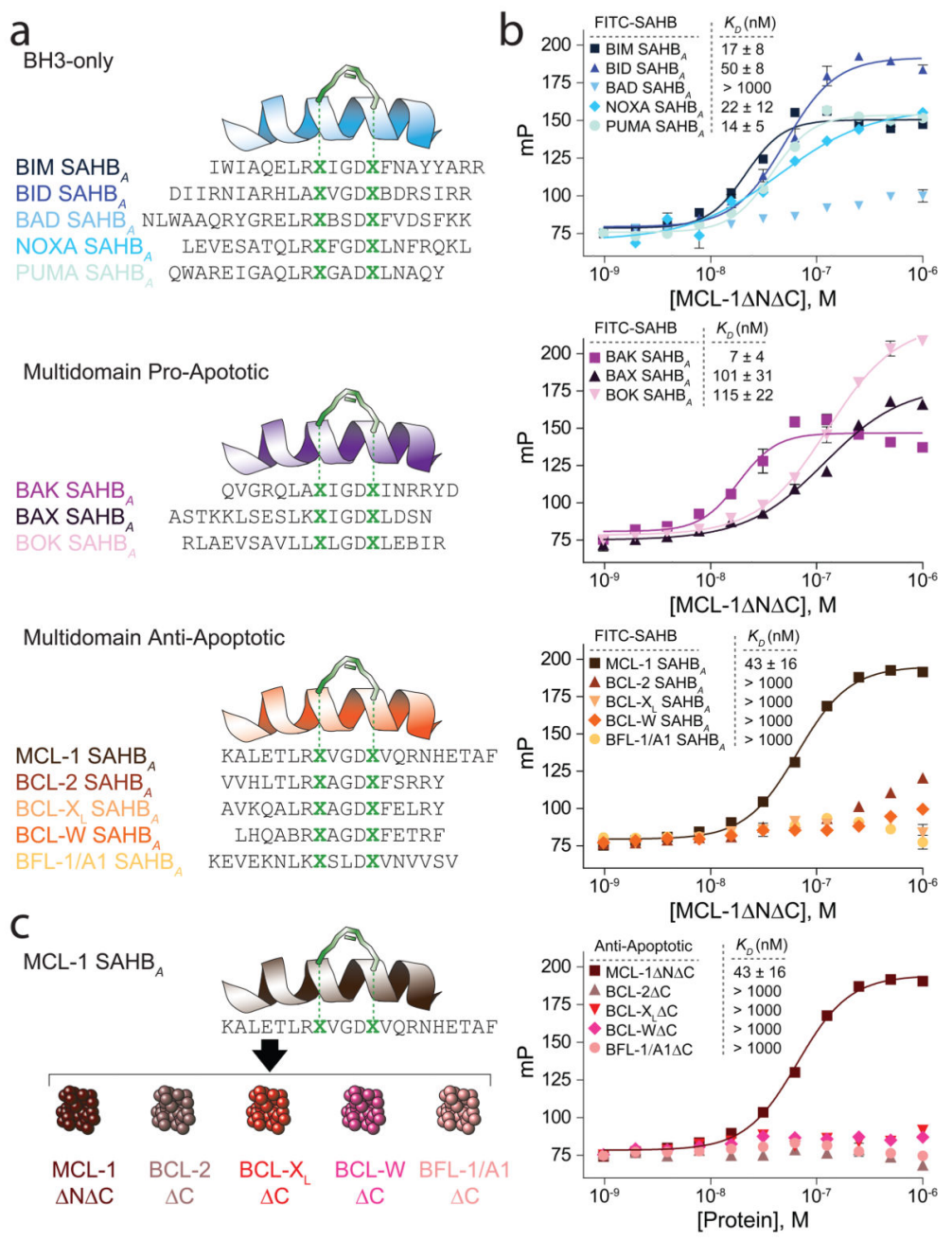

Figure 1.

Identification of an MCL-1-selective BH3 domain. (a) A panel of Stabilized Alpha-Helix of BCL-2 domains (SAHBs) was designed based on the BH3 domains of pro- and antiapoptotic BCL-2 family members. A pair of crosslinking non-natural amino acids (X) were substituted at the indicated $i, i+4$ position of the non-interacting helical surface and "stapled" by ruthenium-catalyzed olefin metathesis. To optimize the activity of the Grubbs' ruthenium catalyst, sulfur-containing methionines were replaced with norleucines, which are designated by the letter B. (b) Dissociation constants for the binding of fluorescently labeled SAHBs to MCL- $1 \Delta \mathrm{N} \Delta \mathrm{C}$ were determined by fluorescence polarization assay (FPA) and nonlinear regression analysis. (c) Among the SAHBs that bound MCL-1 $\Delta \mathrm{N} \Delta \mathrm{C}$ with high affinity, only MCL-1 $\mathrm{SAHB}_{A}$ displayed a potent and exclusive interaction with

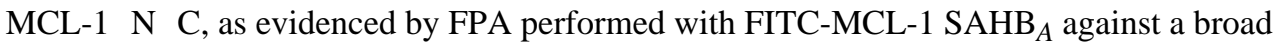
panel of anti-apoptotic targets. Data are mean and s.d. for experiments performed in at least triplicate. 
a

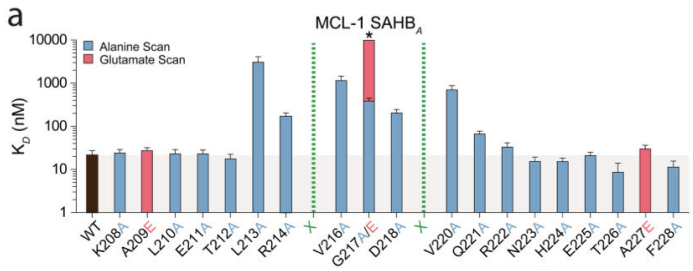

C

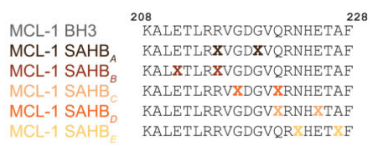

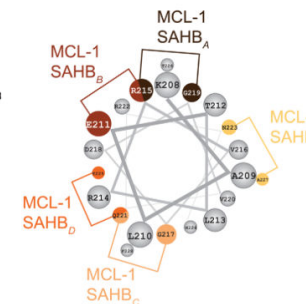

$\mathrm{b}$
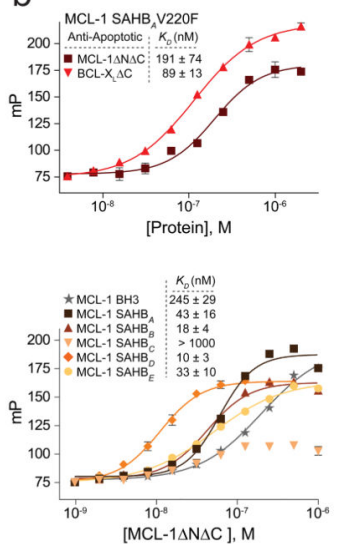

Figure 2.

Binding and specificity determinants of the MCL-1 BH3 helix. (a) A panel of sequential alanine mutants (alanine scan) of FITC-MCL-1 $\mathrm{SAHB}_{A}$ was generated for FPA binding analysis, revealing key residues within the core $\mathrm{BH} 3$ sequence required for high affinity MCL-1 $\Delta \mathrm{N} \Delta \mathrm{C}$ binding. Glutamate mutagenesis was also performed to evaluate the contribution of native alanine and glycine residues to MCL- $1 \Delta \mathrm{N} \Delta \mathrm{C}$ binding. ${ }^{*}, \mathrm{~K}_{D}>10 \mu \mathrm{M}$. (b) A single point mutation of $\mathrm{V} 220 \mathrm{~F}$ eliminated the MCL-1 specificity of MCL-1 SAHB , conferring binding affinity to both MCL- $1 \Delta \mathrm{N} \Delta \mathrm{C}$ and BCL- $\mathrm{X}_{\mathrm{L}} \Delta \mathrm{C}$, as demonstrated by FPA. (c) Sampling a variety of staple positions along the a-helical surface revealed disruption of MCL-1 $\Delta \mathrm{N} \Delta \mathrm{C}$ binding only by the G217,Q221 staple $\left(\mathrm{MCL}-1 \mathrm{SAHB}_{C}\right.$ ), which is located at the hydrophobic binding interface. MCL-1 SAHB ${ }_{D}$ exhibited the strongest binding activity $\left(\mathrm{K}_{D}, 10 \mathrm{nM}\right)$, with 4-fold improvement over the parental MCL-1 SAHB $\mathrm{S}_{A}$. Data are mean and s.d. for experiments performed in at least triplicate. 


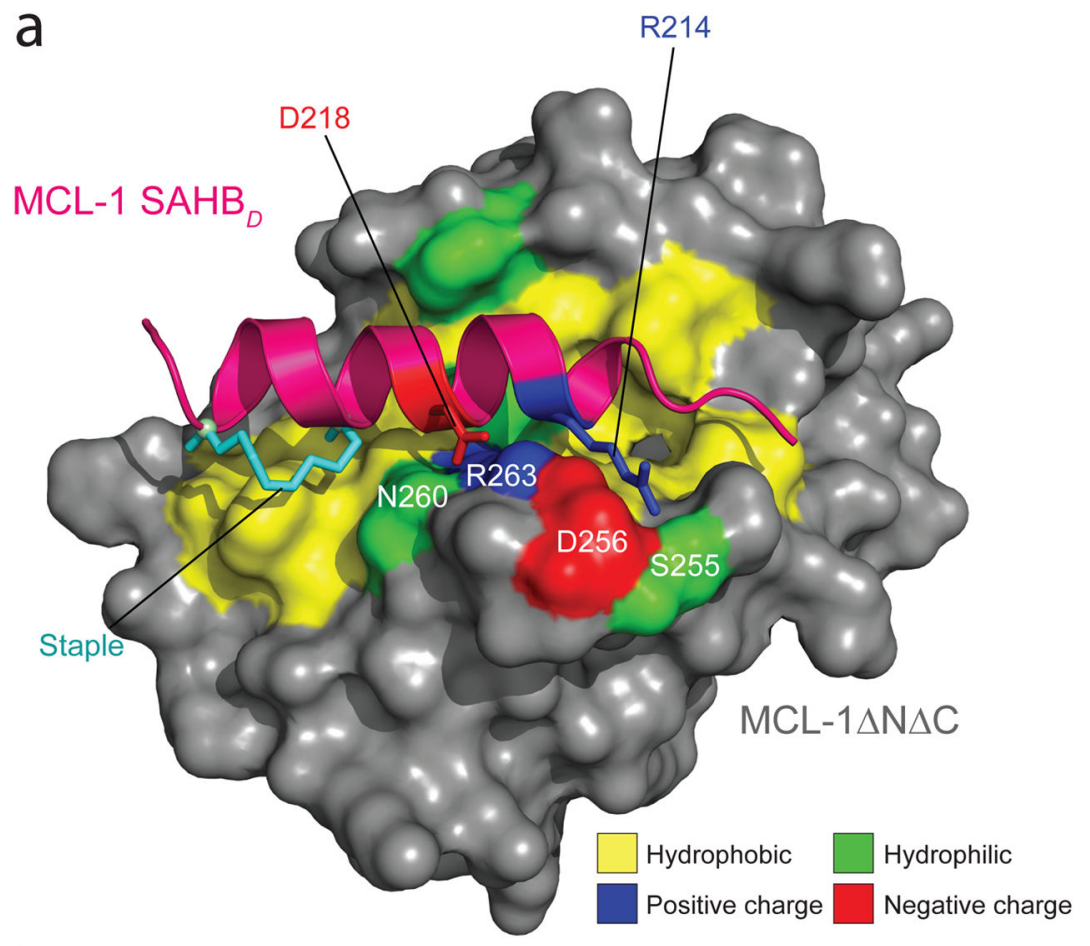

b MCL-1 SAHB

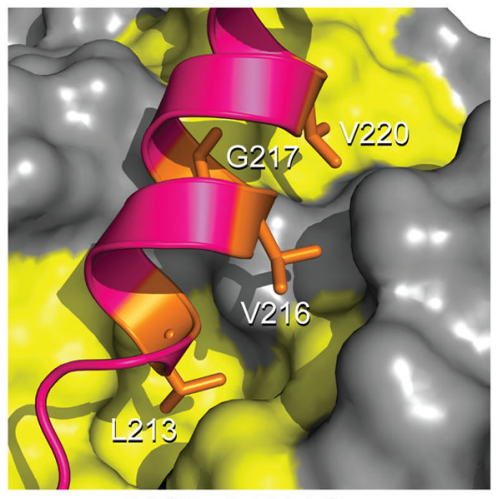

$\mathrm{MCL}-1 \Delta \mathrm{N} \Delta \mathrm{C}$
C MCL-1 SAHB

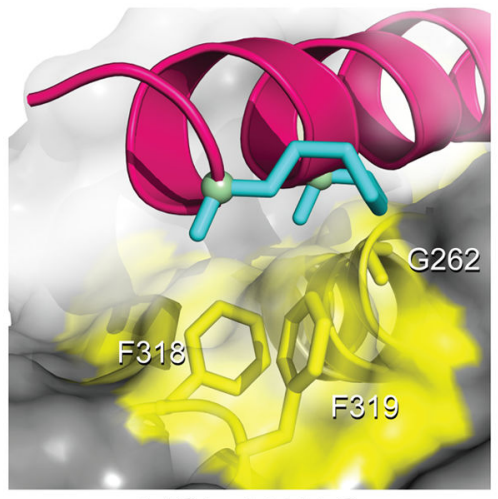

$\mathrm{MCL}-1 \Delta \mathrm{N} \Delta \mathrm{C}$

Figure 3.

Crystal structure of the MCL-1 SAHB ${ }_{D} / \mathrm{MCL}-1 \Delta \mathrm{N} \Delta \mathrm{C}$ complex. (a) MCL-1 SAHB ${ }_{D}$ engages MCL-1 $\triangle \mathrm{N} \Delta \mathrm{C}$ at the canonical $\mathrm{BH} 3$ binding groove of anti-apoptotic proteins, as determined by x-ray crystallography at 2.32- $\mathrm{A}$ resolution (PDB 3MK8). Hydrophobic interactions at the binding interface are reinforced by a complementary polar interaction network that involves MCL-1 SAHB ${ }_{D}$ residues R214 and D218 and MCL-1 $\Delta \mathrm{N} \Delta \mathrm{C}$ residues S255, D256, N260, and R263. The side chains of hydrophobic, positively charged, negatively charged and hydrophilic residues are colored yellow, blue, red and green, respectively. (b) The core BH3 residues L213, V216, G217 and V220 of MCL-1 SAHB ${ }_{D}$ make direct contact with a hydrophobic cleft at the surface of MCL- $1 \Delta \mathrm{N} \Delta \mathrm{C}$. (c) The hydrocarbon staple, bearing an olefin in the cis conformation, contributes additional hydrophobic contacts at the perimeter of the core interaction site. 


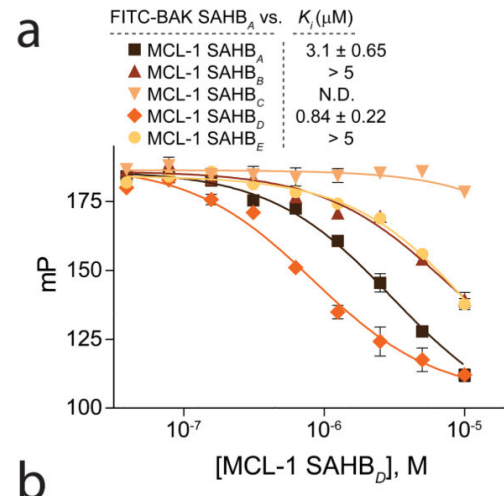

b
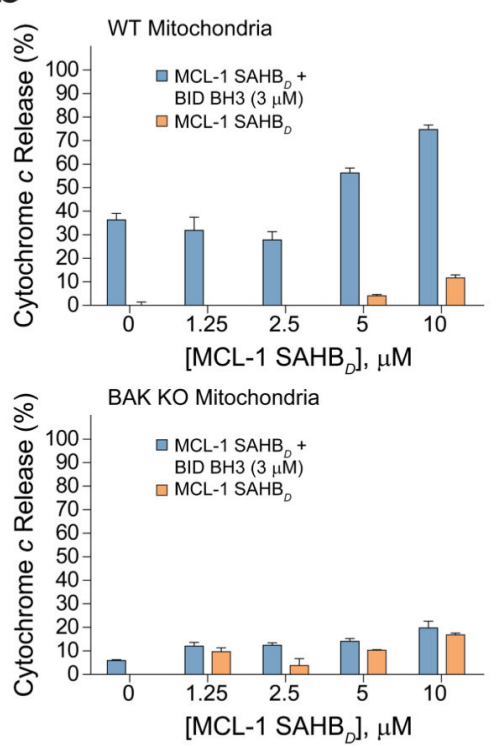

C

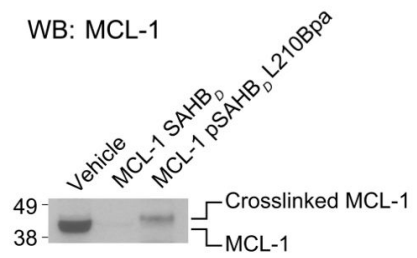

MCL-1 SAHB

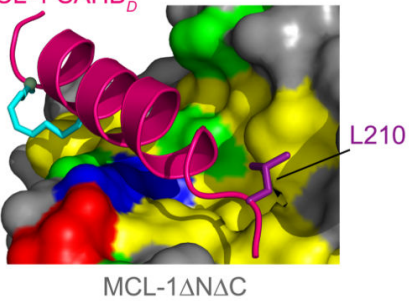

d

WB

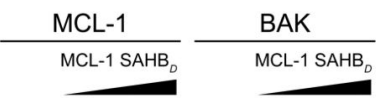

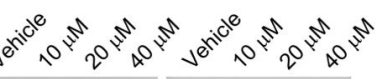

MCL-1 IP

Input

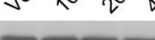

$-=-\frac{10}{20}$

$-$

Figure 4.

MCL-1 SAHB ${ }_{D}$ dissociates the inhibitory MCL-1/BAK complex in vitro and in situ, and sensitizes BAK-dependent mitochondrial cytochrome $c$ release. (a) MCL-1 SAHBs effectively prevent sequestration of the BAK BH3 helix by MCL-1 $\triangle \mathrm{N} \Delta \mathrm{C}$, as demonstrated by competition FPA. N.D., no detected displacement. (b) MCL-1 $\mathrm{SAHB}_{D}$ dose-responsively sensitized BID BH3-induced and BAK-dependent mitochondrial apoptosis, as measured by cytochrome $c$ release assay performed on wild type and $\mathrm{Bak}^{-/-}$mitochondria. (c) An OPM2 multiple myeloma cellular lysate was incubated with the indicated biotinylated MCL-1 $\mathrm{SAHB}_{D}$ constructs in the presence of ultraviolet light, followed by streptavidin-based purification, stringent washing to remove non-covalent binders, elution, and MCL-1 western analysis. The photoreactive MCL-1 $\mathrm{pSAHB}_{D}$, generated by replacing $\mathrm{L} 210$ with a benzophenone-bearing non-natural amino acid (Bpa), directly crosslinked to native MCL-1 within the cellular lysate, whereas no covalent crosslinking was observed for MCL-1 $\mathrm{SAHB}_{D}$, which lacked the photoreactive benzophenone moiety. (d) The native interaction between BAK and MCL-1 was dose-responsively disrupted by treatment of OPM2 cells with MCL-1 $\mathrm{SAHB}_{D}$, as assessed by MCL-1 immunoprecipitation and BAK western analysis. Binding and cytochrome $c$ release data are mean and s.d. for experiments performed in at least triplicate. Vehicle, deionized water. 

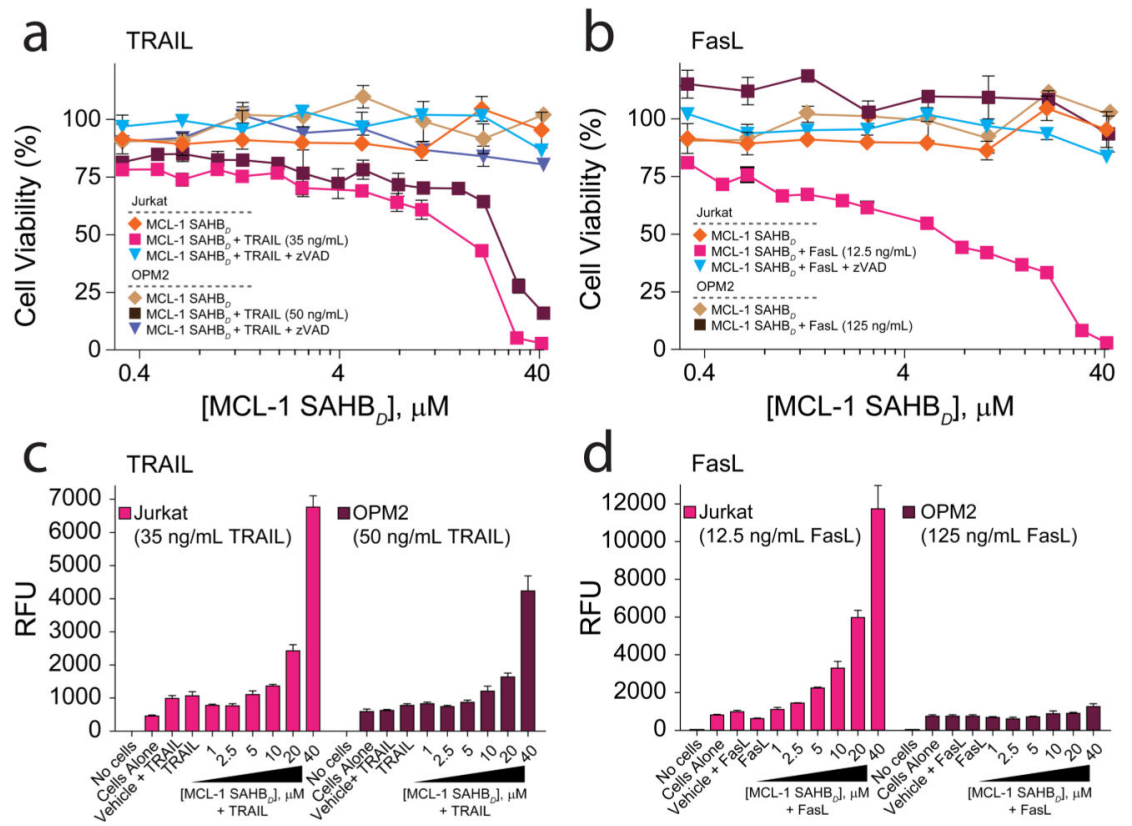

Figure 5.

Selective MCL-1 targeting by MCL-1 SAHB $_{D}$ sensitizes death receptor signaling and induces caspase-dependent cancer cell apoptosis. (a) Jurkat T-cell leukemia and (b) OPM2 cells were exposed to MCL-1 SAHB ${ }_{D}$ singly and in combination with low dose death receptor agonists TRAIL and Fas ligand (FasL) in the presence or absence of the pancaspase inhibitor, z-VAD. Cell viability measured by MTT assay at 24 hours revealed doseresponsive and caspase-dependent apoptosis sensitization of Jurkat (TRAIL and FasL) and OPM2 (TRAIL) cells by MCL-1 SAHB ${ }_{D}$. The capacity of MCL-1 SAHB $D$ to sensitize (c) Jurkat and (d) OPM2 cells to death receptor stimuli correlated with dose-responsive activation of caspase 3/7, as measured by luminescence of DEVD-cleaved substrate. Data are mean and s.d. for experiments performed in at least triplicate. Vehicle, deionized water. 